\title{
SUMOylation of sPRDM16 promotes the progression of acute myeloid leukemia
}

\author{
Song Dong and Jieping Chen ${ }^{*}$
}

\begin{abstract}
Background: In addition to genetic and epigenetic alteration, post-translational modification of proteins plays a critical role in the initiation, progression and maturation of acute myeloid leukemia (AML).

Methods: The SUMOylation site of SPRDM16 at K568 was mutated to arginine by site-directed mutagenesis. THP-1 acute myeloid leukemia cells were transduced with a lentivirus containing wild type or K568 mutant sPRDM16. Proliferation, self-renewal and differentiation of transduced THP-1 cells were analyzed both in vitro cell culture and in mouse xenografts. Gene expression profiles were analyzed by RNA-seq.

Results: Overexpression of sPRDM16 promoted proliferation, enhanced self-renewal capacity, but inhibited differentiation of THP-1 acute myeloid leukemia cells. We further confirmed that K568 is a bona fide SUMOylation site on SPRDM16. Mutation of the sPRDM16 SUMOylation site at K568 partially abolished the capacity of sPRDM16 to promote proliferation and inhibit differentiation of acute myeloid leukemia cells both in vitro and in mouse xenografts. Furthermore, THP-1 cells overexpressing sPRDM16-K568R mutant exhibited a distinct gene expression profile from wild type sPRDM16 following incubation with PMA.
\end{abstract}

Conclusions: Our results suggest that K568 SUMOylation of sPRDM16 plays an important role in the progression of acute myeloid leukemia.

Keywords: SUMOylation, sPRDM16, acute myeloid leukemia

\section{Background}

Acute myeloid leukemia (AML) is a serious disease of the hematopoietic system characterized by de-differentiation and uncontrolled proliferation of immature hematopoietic precursor cells in the bone marrow [1]. Leukemia results from the accumulation of genetic and epigenetic alterations during the multistep process of tumorigenesis, including activation of oncogenes and/or inactivation of tumor suppressor genes. Many transcription factors have been shown to play an important role in aggressive hematological tumors. Within the past decade evidence that post-translational modification of proteins, including phosphorylation [2], acetylation [3], ubiquitination [4] and SUMOylation [5, 6], plays a critical role in the initiation, progression and maturation of AML has accumulated.

Positive regulatory domain I-binding factor 1 and retinoblastoma-interacting zinc finger protein-1 (PRDM16)

\footnotetext{
* Correspondence: chenjpxn@163.com

Department of Hematology, Southwest Hospital, Third Military Medical University, 30 Gaotanyan Street, Chongqing 400038, People's Republic of China
}

is a transcription factor [7]. PRDM16 is also termed MEL1 because it shares $63 \%$ sequence similarity with PRDM3/ MECOM (MDS1 and EVI1 complex) [8]. PRDM16 encodes two isoforms: the full-length PRDM16 (or MEL1) and the short-form sPRDM16 (or MEL1S) [9]. PRDM16, but not sPRDM16, contains a 134-amino acid PR domain, which is highly homologous to the SET domain, a structural hallmark of histone methyltransferases [7, 10]. PRDM16 plays an important role in palatogenesis [11], maintenance of hematopoietic [12] and neuronal stem cells [13], and adipose tissue differentiation [14-17]. It acts as an H3K9me1 methyltransferase, which is required to maintain the integrity of mammalian heterochromatin $[7,9,10,18]$. PRDM16 is reported to contribute to translocation-induced leukemia $[7,9,10,18]$. However, only the PR domainnegative isoform of sPRDM16 is potentially oncogenic in leukemia [10], and the underlying molecular mechanisms are largely unknown.

Small ubiquitin-related modifier (SUMO) is a highly conserved ubiquitin-like protein which acts as a reversible 
and highly dynamic post-translational modifier of a large number of proteins $[19,20]$. SUMOylation is catalyzed by a multistep enzymatic cascade including activating (E1), conjugating (E2), and ligating (E3) enzymes. SUMOylation alters the localization, activity or stability of target proteins [19], and is reversed by a family of Sentrin/SUMO-specific proteases (SENPs).

Accumulating evidence has shown that SUMOylation plays a wide range of roles in the regulation of growth and development of all eukaryotes, for example, influencing transcriptional regulation and genome integrity. Deregulation of SUMOylation has been found in many human diseases including cancer, seizures and Alzheimer's diseases [21]. Recently, sPRDM16 was reported to be SUMOylated [22], and we have found PRDM16 to also be SUMOylated (data not shown). However, the role of PRDM16 SUMOylation in progression of AML is unknown.

In this study, we have explored sPRDM16 SUMOylation in AML in vivo and in vitro. We found that SUMOylation of sPRDM16 regulated expression of genes during AML differentiation, and promoted AML progression while inhibiting differentiation of AML cells.

\section{Methods}

\section{Plasmids and antibodies}

The plasmid MSCV-PRDM16 was purchased from Addgene (Cambridge, MA, USA). Plasmid FLAGsPRDM16 was generated from MSCV-PRDM16 by standard molecular cloning methods. Plasmids HA-SUMO1, HA-UBC9, RGS-SENP1 and RGS-SENP1-mutant were designed and developed as previously described [23]. Anti-Flag antibody was obtained from Sigma (clone M2, St. Louis, MO, USA); anti-HA antibody was purchased from COVANCE (Beijng, China); anti-RGS antibody was obtained from QIAGEN (Germantonw, MD, USA); and anti-SUMO1 and PRDM16 antibodies from Abcam (Cambridge, MA, USA).

\section{Site-directed mutagenesis}

The potential SUMOylation residues from lysine (K) to arginine ( $R$ ) in sPRDM16 were mutated using the QuickChange ${ }^{\mathrm{TM}}$ site-directed mutagenesis kit (Stratagene, La Jolla, CA, USA).

FLAG-sPRDM16-K568R was generated with the following primers:

Sense 5'-TTGCTGGTCAGGGCTGAGCCA -3 ' and Antisense 5' -TGGCTCAGCCCTGACCAGCAA-3' .

\section{Cell culture}

HEK293T cells were cultured in DMEM (Hyclone) supplemented with $10 \%$ fetal bovine serum (Gibco) and $1 \%$ penicillin-streptomycin (Gibco). THP-1 cells were maintained at $37{ }^{\circ} \mathrm{C}$ with $5 \% \mathrm{CO}_{2}$ in RPMI-1640 Medium
(Hyclone) supplemented with $0.05 \mathrm{mM}$ 2-mercaptoethanol (Sigma) and $10 \%$ fetal bovine serum (Gibco). Plasmids were transiently transfected into HEK293T cells using Lipofectamine ${ }^{\mathrm{TM}} 2000$ (Invitrogen) according to manufacturer's instructions.

\section{Evaluation of cell adherence (morphological differentiation)}

Differentiation of THP-1 cells to macrophage-like cells was assessed by measureing adherence to plastic cell culture wells. Log phase cells were centrifuged and resuspended at at $1 \times 10^{6} \mathrm{cell} / \mathrm{ml}$ in fresh RPMI1640 complete medium containing $3 \mathrm{nM}$ PMA and incubated for $24 \mathrm{~h}$. Nonadherent cells were collected from the supernatant after washing, then adherent cells were separated gently by cell scraper on ice. The adherent and non-adherent cells were counted, and the sum correlated well with the original number of cells plated. To evaluate of differentiation, control and treated cells were removed from the Petri dishes, pelleted by centrifugation, and resuspended in $1 \mathrm{ml}$ fresh medium.

\section{Western blot}

Total protein was extracted from cells or tissues using radioimmune precipitation assay (RIPA) buffer $(50 \mathrm{mM}$ Tris- $\mathrm{HCl}$ pH7.4, $150 \mathrm{mM} \mathrm{NaCl}, 1$ \% NP-40, 0.1 \% SDS, $1 \mathrm{mM}$ EDTA) with $1 \%$ protease inhibitor cocktail. Equal amounts of protein extracts $(40 \mu \mathrm{g})$ were separated by $10 \%$ sodium dodecyl sulfate-polyacrylamide gel electrophoresis (SDS-PAGE) and transferred onto a PVDF membrane. Membranes were blocked with $5 \%$ w/v non-fat dry milk dissolved in Tris-buffered saline plus Tween-20 (TBS-T; $0.1 \%$ Tween-20; pH 8.3) at room temperature for $1 \mathrm{~h}$, and incubated with primary antibodies at $4{ }^{\circ} \mathrm{C}$ overnight. After washing with TBS-T, membranes were incubated with horseradish peroxidase (HRP)-labeled secondary antibodies for $1 \mathrm{~h}$ at room temperature. Immunobands were visualized using enhanced chemiluminescence (ECL) kit (GE Healthcare, Waukesha, WI, USA) according to manufacturer's instructions and exposed to $\mathrm{X}$-ray films.

\section{Immunoprecipitation}

Cells were collected at $48 \mathrm{~h}$ after transfection and lysed using an ice-cold RIPA buffer (50 mM Tris- $\mathrm{HCl}$ pH7.4, $150 \mathrm{mM} \mathrm{NaCl}, 1$ \% NP-40, $0.1 \%$ SDS, 1 mM EDTA) with $10 \mathrm{mM}$ N-ethylmaleimide, $1 \mathrm{mM}$ PMSF and protease inhibitors (Roche). Cell lysis was performed on ice for $20 \mathrm{~min}$ and the cell lysate was sonicated for $5 \mathrm{~s}$ three times. After centrifugation at $14,000 \mathrm{~g}$ for $10 \mathrm{~min}$ at $4{ }^{\circ} \mathrm{C}$, the supernatants were added to the appropriate antibody coupled to $20 \mu \mathrm{l}$ of anti-FLAG M2 agarose beads (Sigma). The bead suspension was incubated at $4{ }^{\circ} \mathrm{C}$ for $2 \mathrm{~h}$ on a rotating shaker. Beads were then washed 5 times with 
RIPA buffer, mixed with $15 \mu \mathrm{L} 2 \times$ SDS sample buffer and boiled at $100{ }^{\circ} \mathrm{C}$ for $5 \mathrm{~min}$. The samples were subjected to Western blot analysis.

\section{Lentiviral transduction}

To obtain lentivirus particles, $5 \times 10^{5}$ HEK293T packaging cells were plated in $60-\mathrm{mm}$ culture dishes and transiently transfected with $2 \mu \mathrm{g}$ of each lentivirus vector mixture (pCDH-sPRDM16 $1 \mu \mathrm{g}$, psPAX2 $0.75 \mu \mathrm{g}$, pMD2.G $0.25 \mu \mathrm{g}$ ) together with $5 \mu \mathrm{l}$ Lipofectamine 2000 (Invitrogen). Supernatant containing lentivirus was collected $36 \mathrm{~h}$ after transfection, filtered using $0.45-\mathrm{mm}$ filters and used immediately for infection. Logarithmically growing THP-1 cells were transduced with lentivirus as previously described [24].

\section{Cell proliferation assays}

To assess cellular proliferation, cells were seeded in 24-well plates $\left(2 \times 10^{5}\right.$ cells in $1 \mathrm{ml}$ medium per well $)$ and counted each day using a hematocytometer and trypan blue staining to exclude dead cells. Colorimetric proliferation assays were performed in 96-well plates as 8-fold measurements. A WST-8 [2-(2-methoxy-4nitrophenyl)-3-(4-nitrophenyl)-5-(2,4-disulfophenyl)-2H-tet razolium, monosodium salt] assay (Dojindo, Shanghai, China) was conducted using 2000 cells in $100 \mu \mathrm{l}$ medium per well in 96-well plates. The cell counting kit-8 (CCK-8) assay was performed according to the manufacturers' recommendations.

\section{Soft agar colony formation assay}

A soft agar suspension ( $0.35 \%$ agar) containing colonyforming cells was plated over a soft agar underlay (0.6\% agar). THP-1 cell complete culture medium was added and changed twice a week. After 1000 cells were embedded in the soft agar in 6-well plates (end concentration $0.35 \%$ agar in complete medium) and grown over 12 days, colony numbers were counted under microscope.

\section{Flow cytometry}

For flow cytometry, cells were fixed with $4 \%$ paraformaldehyde in PBS for $10 \mathrm{~min}$ and then blocked with $1 \%$ BSA in PBS for $10 \mathrm{~min}$ at room temperature. Fixed, blocked cells were incubated with APC-conjugated anti-CD11b monoclonal antibody (BD PharMingen) for $15 \mathrm{~min}$ on ice, and cells were washed 3 times with $1 \%$ BSA in PBS. Staining was analyzed using a FACS Calibur instrument (BD PharMingen).

\section{Animal systemic leukemia models}

NOD.CB17-Prkdc ${ }^{\text {scid } / J ~(N O D / S C I D) ~ m i c e ~ w e r e ~ p u r-~}$ chased from Shanghai SLAC laboratory Animal Co., Ltd. (Shanghai, China). Animals were maintained at the animal facility of Shanghai Jiao Tong University School of Medicine in accordance with the local regulations and handled under sterile conditions. The study protocol was approved by the Review Committee for the Use of Human or Animal Subjects of Shanghai Jiao Tong University School of Medicine. Transplantations were performed by intravenous injections of six to eight week old mice. THP-1 cells $\left(1 \times 10^{7}\right.$ cells per mouse in $200 \mathrm{ml}$ saline vehicle) were injected into the tail vein to create the leukemia models $5 \mathrm{~h}$ after total body irradiation with 1.5 Gy using a 137Cs source to enhance angiogenic potential [25]. After transplantation, the mice were monitored for leukemia symptoms, such as weight loss, hunch-back, and decreased activity. All procedures were carried in accordance with national and international laws and policies.

\section{MRNA-sequencing and data analysis}

RNAs from the THP-1 cell line with stable expression of sPRDM16-WT or sPRDM16-K568R or a mocktransfected cell line were purified using Trizol ${ }^{\mathrm{TM}}$ method and subsequently cleaned using RNAeasy Kit (Qiagen). The polyadenylated RNAs purified from the cells were used for the construction of a sequencing library using the ScriptSeq Complete Gold Kit (Epicentre, Illumina). Cluster generation and sequencing were carried out using standard procedures in Hi-Seq 2500 Illumina platform. We used a single-end sequencing protocol to generate a $50 \mathrm{nt}$ read at each end. RNA-seq reads were aligned to the human genome using TopHat (Johns Hopkins University, Baltimore, MD, USA). Cufflinks was employed to normalize Data and perform relevant comparisons among the different samples. Gene ontology analysis was performed using DAVID GO analysis software to search for enriched pathways.

\section{Quantitative real-time PCR analysis}

Total RNA was extracted by Trizol kit (Invitrogen) and treated with DNase (Promega). Complementary DNA was reverse transcribed using M-MLV reverse transcriptase and random hexamers according to the manufacturer's protocol (Takara). All experiments were performed with Power SYBR $^{\circ}$ Green PCR Master Mix (Applied Biosystems) using the LightCycler 480 Real-Time PCR System (Roche). PCR was carried out in triplicate and standard deviations representing experimental errors were calculated. Differences in cDNA input were normalized to GAPDH. All data were analyzed by the LightCycler 480 software (Roche). The following PCR primers were used:

hKLF10-forward-5' -ACTGCGGAGGAAAGAATGGA3', hKLF10-reverse-5' -CTGGGAGGAGTGCTGGGAA C-3'; hCCL5-forward-5' -GCTGTCATCCTCATTGCTAC3', hCCL5-reverse-5'-CATTTCTTCTCTGGGTTGGC-3'; IL6R-forward-5' -TGCCAGTATTCCCAGGAGTC-3', IL6R-reverse-5' -GGCAGTGACTGTGATGTTGG-3'; 


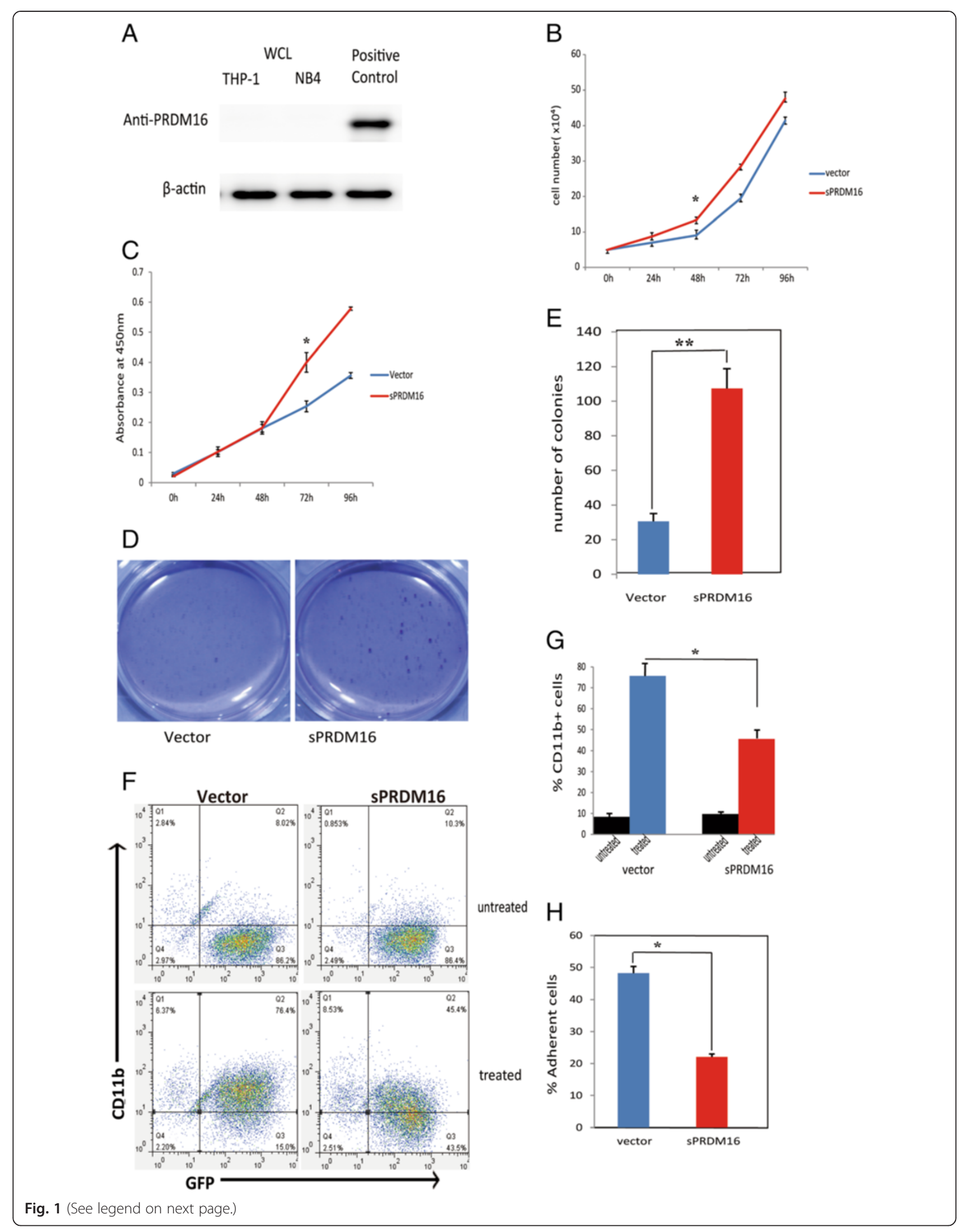


(See figure on previous page.)

Fig. 1 sPRDM16 promoted the proliferation, enhanced the capacity for self-renewal and inhibited the differentiation of acute myeloid leukemia cells. a Protein levels of sPRDM16 in THP-1 and NB4 cell lines. The original whole-cell lysates (WCL) were analyzed by immunoblotting (IB) with anti-PRDM16 or anti-actin antibodies. b Overexpression of sPRDM16 promoted proliferation of THP-1 cells. THP-1 cells stably transfected with either vector (Vector-THP-1) or SPRDM16 (SPRDM16-WT-THP-1) were cultured and cell number was counted at the indicated time. Data are presented as mean \pm S.D. of three independent experiments in RPMI-1640 medium. The number of vector and SPRDM16 differed significantly at $48 \mathrm{~h}(\mathrm{P}<0.01)$. c Proliferation of the THP-1 cells was determined using CCK-8 assay. The absorbance of the CCK- 8 assay solution was detected with plate reader $(450 \mathrm{~nm}$ filter). Data are presented as means \pm S.D. of three independent experiments. Difference between two cell lines was significant at $48 \mathrm{~h}(P<0.01)$. d Overexpression of sPRDM16 increased colony formation of THP-1 cells. THP-1 cells stably transfected with either vector (Vector-THP-1) or sPRDM16 (SPRDM16-WT-THP-1) were seeded in $1 \mathrm{ml}$ of medium containing $10 \%$ FBS with $0.35 \%$ soft agar at $1 \times 10^{3}$ cells per well and layered onto the base soft agar medium. The photographs were taken after 12 days. Images are representative of three independent experiments. e The number of colonies in colony formation assay was scored after 12 days. The colonies were visualized by staining with $0.5 \%$ crystal violet. The experiments were analyzed in triplicate and colonies larger than $50 \mu \mathrm{m}$ in diameter were counted under microscope. Columns, mean of triplicate experiments; bars, S.D. An unpaired (equal variance) t-test was performed to compare sPRDM16-WT and vector control $* * P<0.01)$. f Overexpression of sPRDM16 suppressed differentiation of THP-1 cells. Logarithmically growing cells were exposed to $3 \mathrm{nM}$ PMA for $24 \mathrm{~h}$. The percentage of cells expressing the monocytic maturation marker $\mathrm{CD} 11 \mathrm{~b}$ was determined by flow cytometry. The results are representative of three independent experiments. $\mathbf{g}$ The results of flow cytometry were represented as a histogram. Values represent the mean \pm S.D. for experiments performed in triplicate. ${ }^{*} P<0.01$ represents a significant difference from the cells treated with PMA $(n=3)$; columns, mean of triplicate experiments; bars, S.D. $\mathbf{h}$ The same THP-1 cells were treated as E. Logarithmically growing cells were treated with $3 \mathrm{nM}$ PMA for $24 \mathrm{~h}$. The percentage of adherent cells was calculated. Values represent the mean \pm S.D. for experiments performed in triplicate. ${ }^{*} P<0.01$ represents a significant difference from cells treated with PMA $(n=3)$; columns, mean of triplicate experiments; bars, S.D.

hLIF-forward-5' -ACAGAGCCTTTGCGTGAAAC-3', hLIF-reverse-5'-TGGTCCACACCAGCAGATAA-3'; hNUMB-forward-5'-CGATGACCAAACCAGTGACAG3', hNUMB-reverse-5'-AGAGGGAGTACGTCTATGAC CG-3'; hBCL3-forward-5'-ACTGCCTTTGTACCCCAC TC-3', hBCL3-reverse-5' -GGTATAGGGGTGTAGGCA GGT-3'; hHDAC9-forward-5' -GGATCAAAGCTCTCCA CCCC-3', hHDAC9-reverse-5' -TGGGCTCAGAGGCAG TTTTT-3'.

GAPDH-forward-5' -AGAAGGCTGGGGCTCATTTG -3', GAPHD-reverse-5' -AGGGGCCATCCACAGTCTT C-3'.

Results were expressed as relative expression, normalized to the internal control.

\section{Statistical analysis}

All data are presented as mean \pm standard deviation (S.D.). Statistical analysis was performed using Student's t-test and values of $P \leq 0.05$ were considered statistically significant.

\section{Results}

\section{sPRDM16 promotes proliferation, enhances self-renewal} capacity, while inhibiting differentiation of acute myeloid leukemia cells

Protein expression of sPRDM16 wasn't detected in THP-1 or NB4 cell line (Fig. 1a) and very few leukemic cell lines express SPRDM16 [10]. To investigate the role of sPRDM16 in AML progression, we cloned the DNA fragment from the internal initiation codon ATG597 in exon 4 to exon 17 of PRDM16 into the PCDH lentivirus vector with FLAGtag at the $\mathrm{N}$-terminal, and established stablly infected cell lines. We first monitored the proliferation of THP-1 cells stably transfected with either vector (Vector-THP-1) or sPRDM16 (sPRDM16-WT-THP-1). We found that cells overexpressing sPRDM16 proliferated more rapidly than Vector-THP-1-transfected cells (Fig. 1b). Similar results were obtained by CCK- 8 assay (Fig. 1c).

To assess whether sPRDM16 enhanced self-renewal of leukemia cells, we performed a soft agar colony formation assay in the presence of $10 \% \mathrm{FBS}$ and monitored cell growth. After 12 days, sPRDM16-WT-THP-1 cells had formed more soft agar colonies than Vector-THP-1 cells $(P<0.01)$ (Fig. $1 \mathrm{~d}$ and e.

To determine whether sPRDM16 played a role in the maturation of AML cells, we incubated THP-1 cells with 3 nM PMA for $24 \mathrm{~h}$, and measured cell-surface expression of the monocytic maturation marker CD11b using APClabeled anti-CD11b antibody by flow cytometry (Fig. 1f). Expression of CD11b did not differ significantly between Vector-THP-1 (8.02 \%) and sPRDM16-WT-THP-1 (10.3\%) cells. PMA induced expression of CD11b in $45.4 \%$ of sPRDM16-WT-THP-1 cells, while a significant increase in CD11b expression was observed in Vector-THP-1 cells (76.4 \%, P < 0.01) (Fig. 1f and g). Further, when cell adherence was monitored, as an indication of monocyte differentiation, sPRDM16-WT-THP-1 cells demonstrated less adherence (Fig. 1h).

These results indicate that sPRDM16 promoted proliferation and enhanced self-renewal capacity, while inhibing cellular differentiation in AML, suggesting that sPRDM16 may be an oncogene.

\section{K568 is a bona fide SUMOylation site of sPRDM16}

Overexpression of sPRDM16 with loss of p53 induces myeloid leukemia in mice [10]. It was also reported that SUMOylation of sPRDM16 leads to its interaction with $\mathrm{CtBP}$, facilitating the repressor activity of $\mathrm{CtBP}$ and blockade of G-CSF-induced myeloid differentiation in L-G3 
cells [22]. Thus, we speculated that sPRDM16 SUMOylation may contribute to AML progression. To test this hypothesis, we first confirmed that sPRDM16 was SUMOylated by SUMO1. We co-transfected HEK293T cells with Flag-sPRDM16, HA-SUMO1 and HA-UBC9 plasmids, and performed co-immunoprecipitation assay. As show in Fig. 2a, sPRDM16 was conjugated to SUMO1. Next, we co-expressed Flag-sPRDM16, HA-SUMO1 and SENP1 in HEK293T cells. SUMOylated sPRDM16 was readily detected when Flag-sPRDM16 and HA-SUMO1 were co-transfected. In sharp contrast, the SUMOylated band disappeared when wild type SENP1 was over- expressed, but not when the catalytic mutant SENP1m was expressed (Fig. 2b). To confirm that the SUMO acceptor site of sPRDM16 was K568, we generated sPRDM16 SUMOylation mutant, sPRDM16-K568R and performed SUMOylation assays in HEK293T cells co-transfected with wild-type sPRDM16-WT or mutant sPRDM16-K568R and HA-SUMO1. As expected, one band of SUMOylated FlagsPRDM16 was observed in the immunoprecipitates of cells transfected with sPRDM16-WT. In contrast, the mutant sPRDM16-K568R completely abolished SUMOylation (Fig. 2c), consistent with the hypothesis that $\mathrm{K} 568$ is a bona fide SUMOylation site of sPRDM16 [22].

\section{A}
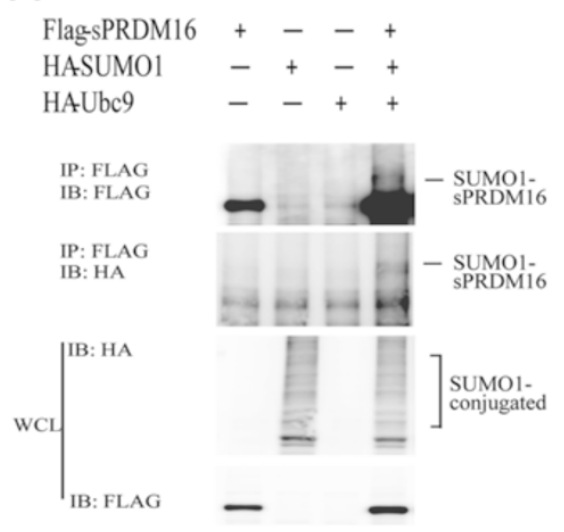

B

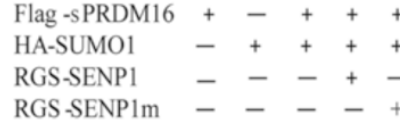

$$
\text { IP: FLAG }
$$
IB: FLAG

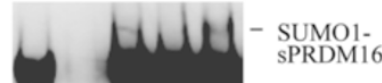

$$
\text { IP: FLAG }
$$
IB: HA

WCL

IB: HA

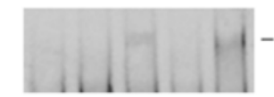
- SUMO1SPRDM16 $\mid \begin{aligned} & \text { IB: FLAG } \\ & \text { IB:RGS }\end{aligned}$

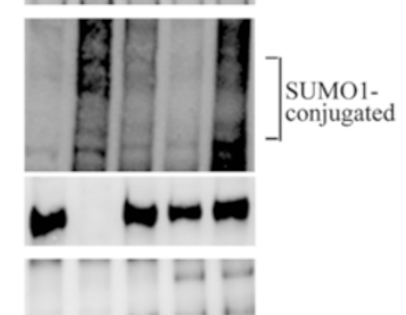

C

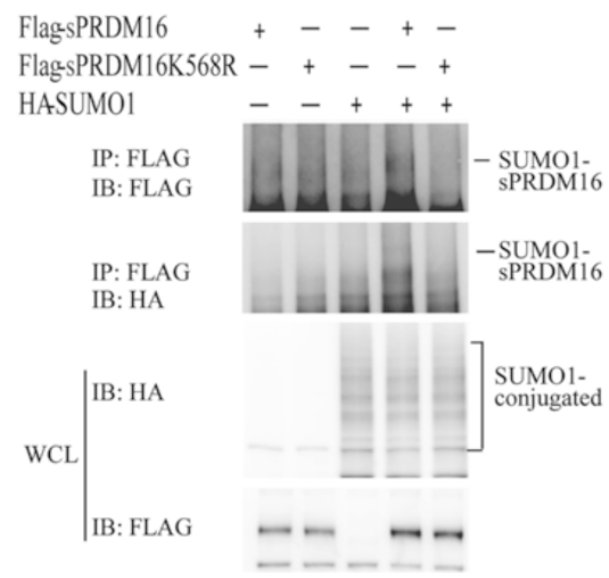

Fig. 2 SPRDM16 was SUMOylated by SUMO1 on lysine-568. a SPRDM16 was SUMOylated in vivo. HEK293T cells were transfected with the indicated plasmids for $36 \mathrm{~h}$. Immunoprecipitation was performed with anti-FLAG M2 agarose beads. The immunoprecipitates (IP) and the original whole-cell lysates (WCL) were analyzed by immunoblotting (IB) with anti-HA or anti-FLAG antibodies. b SENP1 de-SUMOylated sPRDM16. HEK293T cells were transfected with HA-SUMO1, Flag-SPRDM16, RGS-SENP1, or RGS-SENP1m as indicated. Flag-SPRDM16 proteins were pulled down by anti-Flag M2 agarose beads from cell lysates. Bound proteins were blotted with anti-Flag. Cell lysate was immunoblotted (IB) with anti-Flag antibody, anti-HA antibody, or anti-RGS antibody. c K568 was the primary SUMOylation site of sPRDM16. HEK293T cells were transfected with the indicated plasmids. Cell lysates were immunoprecipitated with anti-FLAG M2 agarose beads, followed by Western blot (WB) analysis using anti-HA or anti-FLAG antibodies 


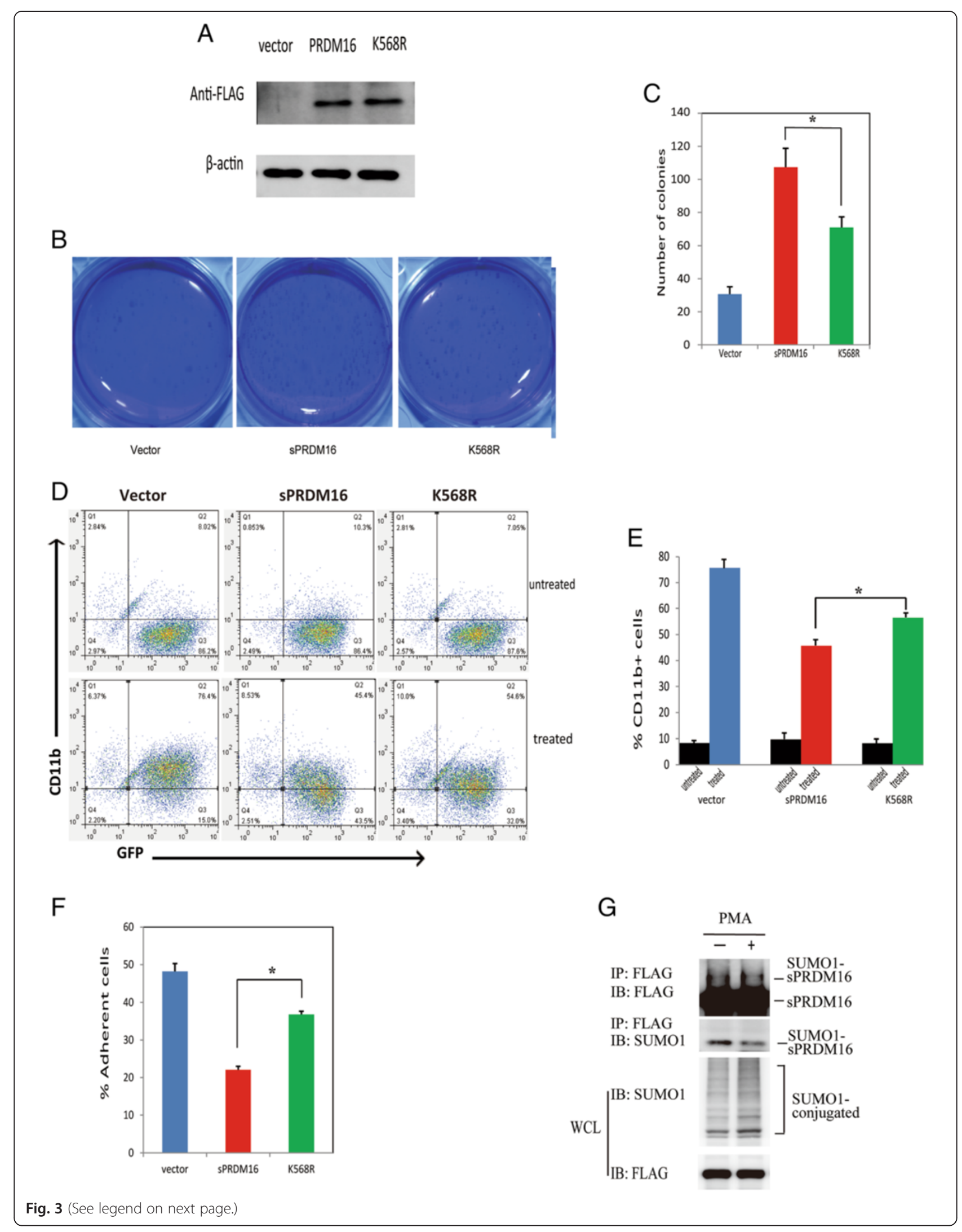


(See figure on previous page.)

Fig. 3 SUMOylation was required for sPRDM16 in the progression of acute myeloid leukemia. a Exogenous protein expression of sPRDM16 or K568R in THP-1 cell line. The original whole-cell lysates (WCL) were analyzed by immunoblotting (IB) with anti-FLAG or anti-actin antibodies. b THP-1 cells stably transfected with either vector, SPRDM16 or sPRDM16-K568R were seeded in $1 \mathrm{ml}$ of medium containing $10 \%$ FBS with $0.35 \%$ soft agar at $1 \times 10^{3}$ cells per well and layered onto the base soft agar medium. Photographs were taken after 12 days. Images are representative of three independent experiments. $\mathbf{c}$ The number of colonies of colony formation assay (A) was scored after 12 days. The colonies were visualized by staining with $0.5 \%$ crystal violet. The experiments were analyzed in triplicate and colonies larger than $50 \mu \mathrm{m}$ in diameter were counted under microscope. Columns, mean of triplicate experiments; bars, S.D. ( $\left.{ }^{*} P<0.01\right)$. d Logarithmically growing THP-1 cells stably transfected with either sPRDM16 or sPRDM16-K568R vector, were exposed to $3 \mathrm{nM}$ PMA for $24 \mathrm{~h}$. The percentage of cells expressing the monocytic maturation marker CD11b was determined by flow cytometry. The dot plot results are representative of three independent experiments. e The results of flow cytometry described in Fig. $3 \mathrm{C}$ were represented as a histogram. Values represent the mean \pm S.D. for experiments performed in triplicate. ${ }^{*} P<0.01$ represents a significant difference from the cells treated with PMA ( $n=3)$; columns, mean of triplicate experiments; bars, s.d. f Logarithmically growing THP-1 cells were treated with 3 nM PMA for $24 \mathrm{~h}$. The percentages of adherence cells on the surface of plastic dish were counted. Values represent the mean \pm S.D. for experiments performed in triplicate. ${ }^{*} P<0.01$ represents a significant difference from the cells treated with PMA $(n=3)$. Columns, mean of triplicate experiments; bars, S.D. $\mathbf{g}$ sPRDM16 SUMOylation in THP-1 cells stably expressing sPRDM16-WT was decreased after incubation with PMA. SUMO1-conjugated proteins in THP-1 cells in the presence or absence of PMA for $24 \mathrm{~h}$ were immunoprecipitated with anti-FLAG M2 agarose beads. SUMO1-sPRDM16 proteins were blotted with anti-FLAG and anti-SUMO1. Cell lysate was immunoblotted with anti-FLAG and anti-FLAG antibody

\section{SUMOylation contributes to SPRDM16-mediated tumorigenesis in acute myeloid leukemia}

To explore the function of sPRDM16 SUMOylation in progression of AML, we generated stable THP-1 cell lines by polyclonal lentiviral infections with Lenti-Vector, sPRDM16-WT or sPRDM16-K568R. Exogenous protein level of sPRDM16 or K568R in THP-1 cell line was shown in (Fig. 3a). We first performed a soft agar colony-forming assay in the presence of $10 \%$ FBS. sPRDM16-WT-THP-1 and sPRDM16-K568R-THP-1 cells formed more numerous and larger colonies than Lenti-Vector transfected cells (Fig. 3b and c). However, colony formation was slower in sPRDM16-K568R transfected cells than sPRDM16-WTTHP-1, suggesting that sPRDM16-K568R reduced the capacity of self-renewal (Fig. 3b and c).

To determine whether SUMOylation of sPRDM16 was involved in differentiation of AML cells, we treated cells stably transfected with Lenti-Vector, sPRDM16-WT and sPRDM16-K568R with $3 \mathrm{nM}$ PMA for $24 \mathrm{~h}$ and measured cell surface expression of the monocytic maturation marker CD11b by flow cytometry. Incubation with PMA significantly increased expression of CD11b in Vector-THP-1, sPRDM16-WT and sPRDM16-K568R-THP-1 cells. However, PMA stimulated CD11b expression less potently in sPRDM16-WT-THP-1 cells (45.4 \%) than sPRDM16K568R cells (54.6 \%) (Fig. 3d and e). Similarly, when cell adherence was monitored, as a marker of monocyte differentiation, sPRDM16-WT-THP-1 cells adhered less well than sPRDM16-K568R cells (Fig. 3f). Meanwhile, sPRDM16 SUMOylation was decreased in SPRDM16-WTTHP-1 cells after incubation with PMA (Fig. 3g).

These results suggest that mutation of sPRDM16 SUMOylation site at K568 reduced the capacity of sPRDM16 to induce proliferation and inhibit differentiation of AML cells, suggesting that K568 SUMOylation of sPRDM16 played an important role in the pathogenesis of AML.
SUMOylation of sPRDM16 enhances the engraftment of systemic THP-1 transplantation leukemia in NOD.CB17-Prkdc ${ }^{\text {scid/J }}$ (NOD/SCID) mice

To confirm our observations in vivo, we transplanted sub-lethally irradiated mice with $1 \times 10^{7}$ GFP-labeled THP-1 cells stably infected with Lenti-Vector, sPRDM16WT or sPRDM16-K568R. The fluorescent disseminated leukemia grafts were monitored by flow cytometry for 30 days post-transplantation. As shown in Figs. 4a and b, in bone marrow fractions, the frequency of GFP + cells in the Lenti-Vector-transplanted group was lower than in either sPRDM16-WT-transplanted or sPRDM16-K568Rtransplanted animals. However, the frequency of GFP + cells in the sPRDM16-WT-transplanted groups was higher than in the sPRDM16-K568R-transplanted group. Similar results were observed in analysis of mouse peripheral blood (Fig. 4c and d). To investigate the engraftment of leukemic cells in bone marrow, we subsequently sectioned the bone marrow tissues and conducted hematoxylin and eosin (H\&E) staining (Fig. 4e). We also continuously monitored the body weight of mice. Consistent with the flow cytometry results, sPRDM16-WT-transplanted animals lost more weight than sPRDM16-K568R-transplanted and the Lenti-Vector-transplanted animals (Fig. 4f). But the difference was not statistically significant. Taken together, these results indicate that sPRDM16 SUMOylation enhanced engraftment of systemic THP-1 transplantation leukemia in NOD.CB17-Prkdc ${ }^{\text {scid } / J ~(N O D / S C I D) ~ m i c e, ~}$ suggesting that mutation of sPRDM16 K568R partially attenuates the progression of AML in vivo.

\section{Differentiation-related genes induced by PMA are differentially expressed between THP-1 cells stably expressing sPRDM16-WT and sPRDM16-K568R}

To further investigate the physiological significance of sPRDM16 SUMOylation, we conducted high-throughput sequencing of mRNA (mRNA-seq) with THP-1 cells 


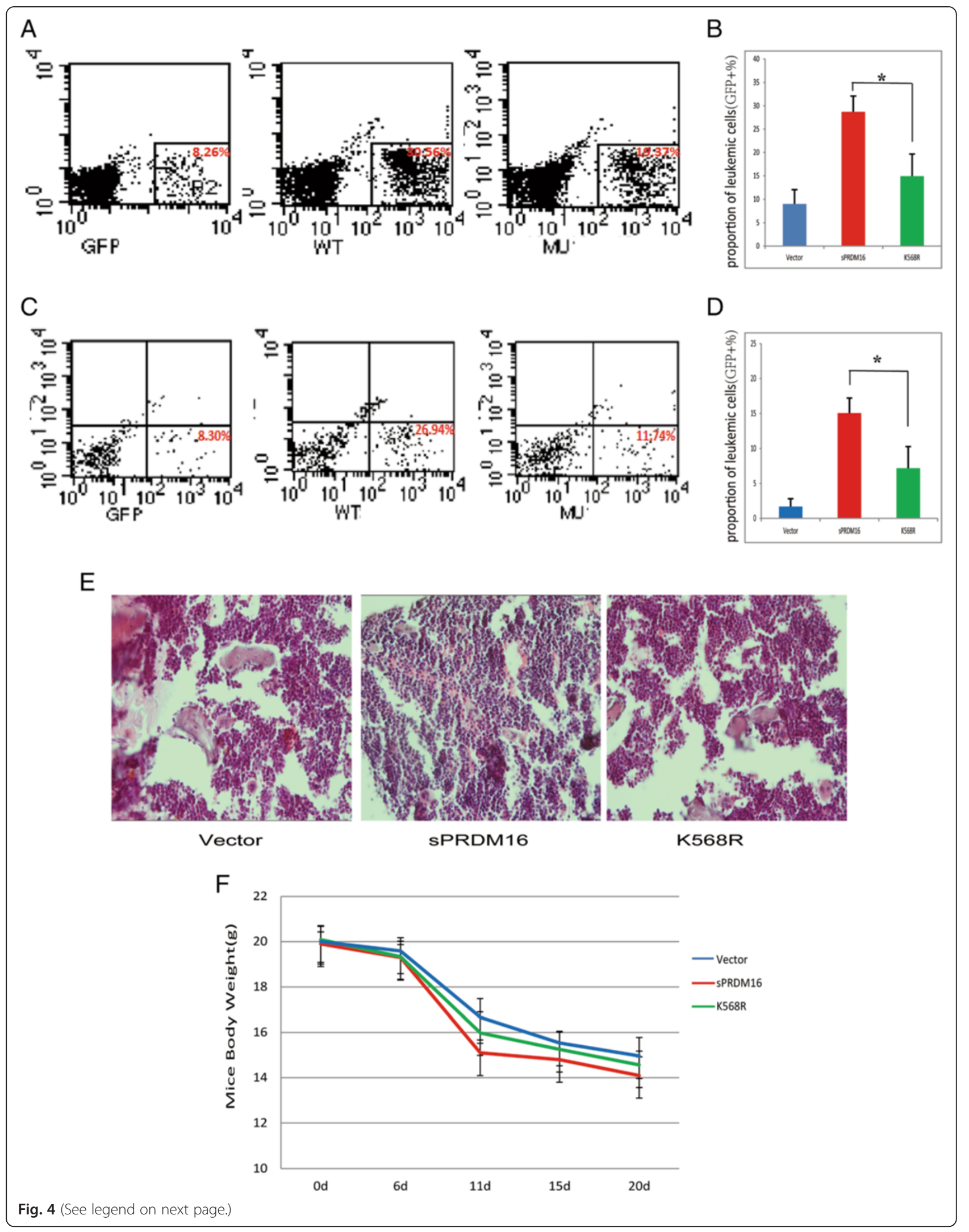


(See figure on previous page.)

Fig. 4 SUMOylation of sPRDM16 enhanced the engraftment of systemic THP-1 transplantation leukemia in NOD.CB17-Prkdc ${ }^{\text {scid } / J ~(N O D / S C I D) ~ m i c e . ~}$ Sub-lethally irradiated mice were inoculated via the tail vein with $1 \times 10^{7}$ GFP-labeled THP-1 cells stably infected with Lenti-Vector, sPRDM16-WT or sPRDM16-K568R. Fluorescent grafts were monitored by flow cytometry. All mice were sacrificed and subjected to subsequent test 30 days post-transplantation of THP-1 cells. a FACS blots of leukemic cells (GFP+) in the femur bone marrow in mice inoculated with Lenti-Vector, sPRDM16-WT or sPRDM16-K568R THP-1 cells 30 days post-transplantation. The dot plots showed SSC (y axis) versus GFP intensity ( $x$ axis). b Bar graphs summarized the percentage of leukemic cell (GFP+) in the bone marrow per femur over whole population $(n=5-6)$. c Dot blots indicated leukemic cells (GFP+) in mice peripheral blood 30 days post-transplantation of Lenti-Vector, sPRDM16-WT or sPRDM16-K568R THP-1 cells. The dot plots showed SSC ( $y$ axis) versus GFP intensity ( $x$ axis). $\mathbf{d}$ Percentages of leukemic cells (GFP+) in mice peripheral blood over whole population $(n=5-6)$. e H\&E staining of marrow cavity of femur section from the mice transplanted with THP-1 cells stably infected with Lenti-Vector, SPRDM16-WT or sPRDM16-K568R. f Mean animal weight

expressing sPRDM16-WT and sPRDM16-K568R. We analyzed the impact of sPRDM16 SUMOylation global gene expression induced in leukemia cell differentiation using gene ontology. From 10 million 50 bp single-end reads, we identified 237 genes the expression of which differed significantly between sPRDM16-WT-THP-1 and sPRDM16-K568R-THP-1 cells after incubation with PMA (>1.5-fold with adjusted $P<0.05$ ). Based on gene ontology results, sPRDM16 SUMOylation significantly affected the expression of cancer-related genes, including genes implicated in wound response, cell proliferation, chemotaxis, differentiation, and cell cycle progression (Fig. 5a). The findings suggest that SUMOylation of sPRDM16 was involved in cancer cell proliferation and differentiation. Such functional enrichment is not surprising as sPRDM16 is a transcriptional regulator of proliferation and differentiation of hematopoietic cell and adipose differentiation.

We next summarized two clusters of gene annotation and selected 13 genes involved in hematopoietic cell proliferation and differentiation (Fig. 5b). We subsequently validated the mRNA-seq results by measuring the changes in 7 newly identified targets, which were previously implicated in hematopoietic cell proliferation and differentiation. The selected targets of sPRDM16 SUMOylation were further validated by real-time RTPCRs. The RT-PCR results were highly correlated with those observed with mRNA-seq (Fig. 5c). These results demonstrated that SUMOylation of sPRDM16 controls the expression of genes related to cancer proliferation and differentiation, suggesting that SUMOylation of sPRDM16 plays an important role in leukemia cell proliferation and differentiation.

\section{Discussion}

In this study, we demonstrated that sPRDM16 promoted the proliferation and enhanced the self-renewal capacity, of THP-1, while inhibiting differentiation of these AML cells. We further confirmed that $\mathrm{K} 568$ is a bona fide sPRDM16 SUMOylation site. Accordingly, mutation of sPRDM16 SUMOylation site K568 partially abolished the influence of sPRDM16 on proliferation and differentiation of AML in vitro and in vivo. Furthermore, THP-1 cells overexpressing sPRDM16-K568R mutant displayed distinct a gene expression profile from wild type sPRDM16 following incubation with PMA. Our findings suggest that K568 SUMOylation plays an important role in the pathogenesis of AML.

PRDM16 has previously been reported to be involved in myeloid and lymphoid malignancies, and to play a role in the regulation of hematopoietic [12], neuronal stem cell growth [13], and differentiation of adipose tissue $[14,17,26]$. It is widely believed that sPRDM16 is an oncogene [27, 28]. Further, it is well documented that sPRDM16 promotes AML progression by regulating gene transcription through direct DNA binding and/or interaction with transcriptional co-factors and chromatin modifiers [29]. Accumulating clinical evidence indicates that deregulation of sPRDM16 is closely associated with abnormal AML phenotypes [9, 10, 28, 30], implicating SPRDM16 in AML pathogenesis. Consistent with these findings, we found that sPRDM16 promoted proliferation and enhanced self-renewal capacity, while inhibiting differentiation of THP-1 AML cells.

In addition, increasing evidence suggest that SUMOylation may have a major role in the evolution of the hematopoietic system and AML. Some studies have indicated that SUMO is an integral component of chromatin and regulates specific transcriptional programs [31]. Recently, Guillaume Bossis et al. reported that an important role of SUMOylation is to regulate the expression of specific genes involved in AML cell response to chemotherapeutic drugs. and inhibition of the SUMO pathway reduces AML cell growth in xenograft mice [6]. The transcriptional activity of sPRDM3, a member of the PR domain family, is negatively regulated by SUMO1 in acute promyelocytic leukemia (APL) [32]. Using various functional assays to compare overexpression of PRDM16-WT or sPRDM16-K568R in THP-1 cells, we demonstrated that sPRDM16 SUMOylation contributed to progression of AML. Furthermore, a SUMOylation mutant of sPRDM16 attenuated its ability to facilitate tumor growth and suppress the differentiation of THP-1 cells in vitro. Animal systemic leukemia transplantation models further indicated that sPRDM16 SUMOylation may be a risk factor for leukemia in vivo. 


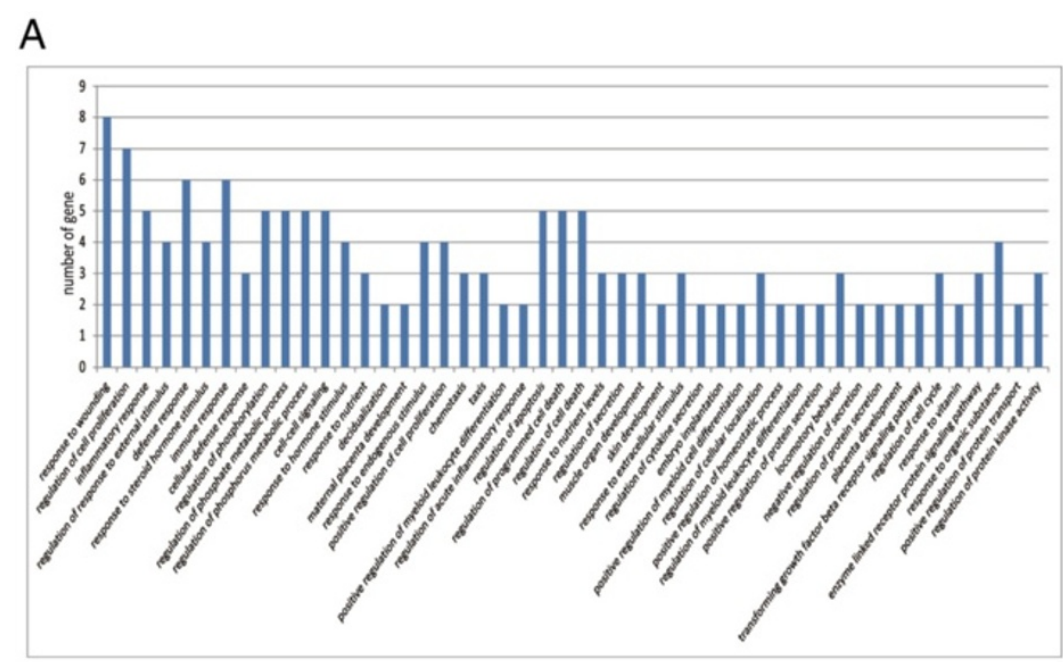

B

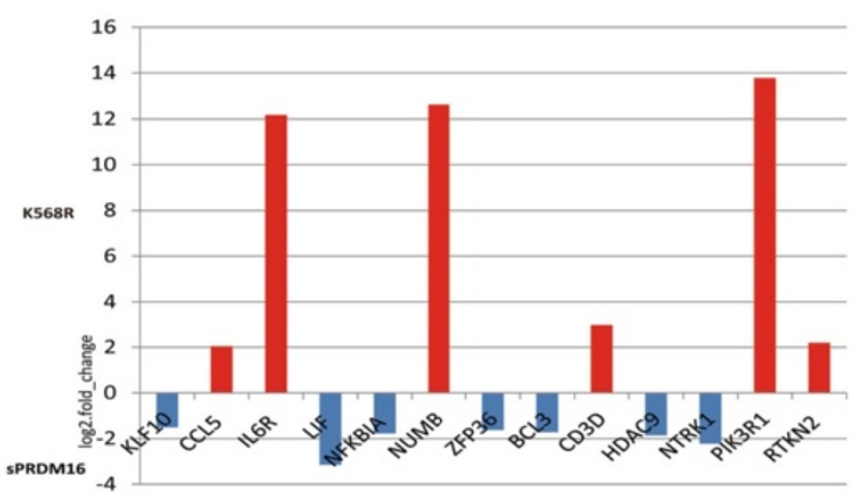

C
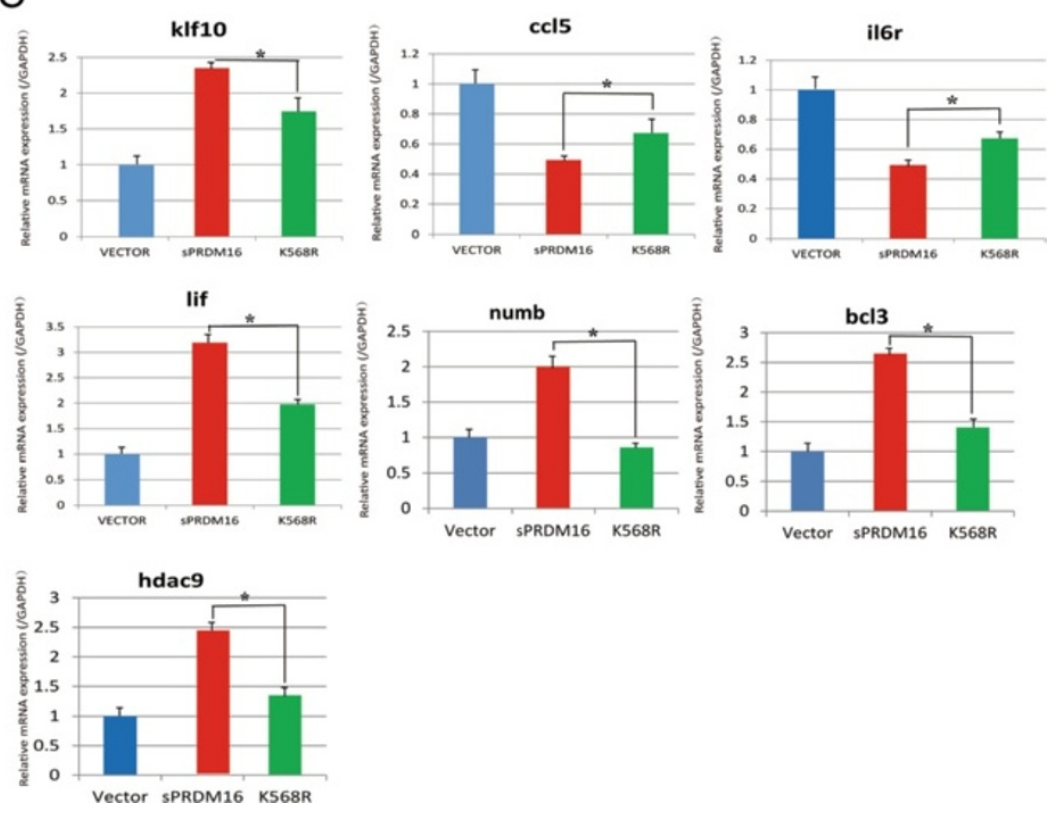

Fig. 5 (See legend on next page.) 
(See figure on previous page.)

Fig. 5 Differentially expressed genes in PMA-induced gene differentiation in THP-1 cells stably expressing sPRDM16-WT and sPRDM16-K568R. a Functional annotation clustering (GO) of genes that were associated with SUMOylation of SPRDM16 in SPRDM16-WT and SPRDM16-K568R-THP-1 cells following incubation with PMA (3 nM/24 h; Analyzed by DAVID, grouped based on the biological process of level 1). b Differential gene expression between sPRDM16-WT and SPRDM16-K568R-THP-1 cells in mRNA-sequencing. $\mathbf{c}$ Expression of differentiation-related genes induced by PMA in THP-1 cells stably expressing SPRDM16-WT and sPRDM16-K568R (including control THP-1 cells with Lenti-Vector). KLF10, CCL5, IL6R, LIF, NUMB, BCL3 and HDAC9 was measured in Lenti-Vector, sPRDM16-WT and sPRDM16-K568R cells by Q-PCR. Data are presented as the $\triangle \mathrm{Ct}$ between the gene of interest and the GAPDH levels expressed in the same sPRDM16-WT-transduced sample or sPRDM16-K568R-transduced sample, relative to the $\triangle \mathrm{Ct}$ observed in the control sample. Mean from three independent experiments are depicted with S.D.

Despite the established role of sPRDM16 in leukemia development, the molecular mechanisms underlying sPRDM16 SUMOylation-mediated progression of AML remain elusive. In particular, very few downstream target genes of sPRDM16 have been identified. Our analysis of stable THP-1 cell lines generated by polyclonal lentiviral infection with sPRDM16-WT or sPRDM16-K568R revealed a distinct gene expression profile. mRNAsequence data indicated no significant difference in gene expression between sPRDM16-WT and sPRDM16K568R-THP-1 cells in the absence of PMA. Interestingly, following induction of differentiation by PMA, we found that 237 genes were differently expressed between sPRDM16-WT and sPRDM16-K568R-THP-1 cells. Consequently, we confirmed that KLF10, BCL3, HDAC9, CCL5, IL6R, LIF and NUMB, all of which are closely related to differentiation in hematopoietic and leukemic cells [33-37], are downstream targets of sPRDM16, and are influenced by SUMOylation. KLF10 is a transcription factor that regulates differentiation of bone marrowderived macrophages $[33,38]$. BCL3 plays a critical role in targeting the differentiation of myeloid progenitors [34]. The chemokine CCL5 induces selective migration of monocytes and drives their differentiation [36]. Numb plays critical roles in cell fate determination as an evolutionary conserved protein $[37,39]$. These results indicate that SUMOylation of sPRDM16 is an important mechanism by which sPRDM16 promotes the growth and proliferation of hematopoietic progenitors and leukemic cells.

\section{Conclusions}

In summary, we have provided evidence that sPRDM16 SUMOylation plays an important role during AML progression by promoting the growth and inhibiting differentiation of AML cells in vitro and in vivo. We further identified significant changes in downstream genes related to SUMOylation of sPRDM16 during AML differentiation. As sPRDM16 is frequently overexpressed in a variety of leukemias, sPRDM16 SUMOylation may be an important regulator of sPRDM16 in leukemia development.

\section{Competing interest}

We have no conflict of interest to declare.

\section{Authors' contributions}

SD carried out all the molecular biology, biochemistry and animal studies, participated in the sequence alignment, performed the statistical analysis and drafted the manuscript. JC conceived of the study, participated in the design and coordination, and helped to draft the manuscript. Both authors read and approved the final manuscript.

\section{Acknowledgments}

This work was funded by grants from National Natural Science Foundation of China (NSFC 39870046, 81270605, 30971066, 81470324), Third Military Medical University Clinical and Science Great Fund Project (2012XLC03), Chongqing Postgraduate Education Reform Project (yjg123114), Chongqing Natural Science Fund Project (CSTC'2008BA5001), The Military Emphasis Medical Scientific Research Project Fund (BWS13C018) and Third Military Medical University Education Reform Project.

Received: 30 May 2015 Accepted: 23 October 2015

Published online: 11 November 2015

\section{References}

1. Zhang H, Alberich-Jorda M, Amabile G, Yang H, Staber PB, DiRuscio A, et al. Sox4 is a key oncogenic target in C/EBPalpha mutant acute myeloid leukemia. Cancer Cell. 2013;24(5):575-88.

2. de la Cruz-Herrera CF, Campagna M, Lang V, del Carmen GonzálezSantamaría J, Marcos-Villar1 J, Rodríguez MS, et al. SUMOylation regulates AKT1 activity. Oncogene. 2015;34(11):1442-50.

3. Shimahara A, Yamakawa N, Nishikata I, Morishita K. Acetylation of lysine 564 adjacent to the C-terminal binding protein-binding motif in EVI1 is crucial for transcriptional activation of GATA2. J Biol Chem. 2010;285(22):16967-77.

4. Tatham MH, Geoffroy M-C, Shen L, Plechanovova A, Hattersley N, Jaffray EG, et al. RNF4 is a poly-SUMO-specific E3 ubiquitin ligase required for arsenicinduced PML degradation. Nat Cell Biol. 2008;10(5):538-46.

5. Ivanschitz1 L, DeThé H, Le Bras M. PML, SUMOylation, and Senescence. Front Oncol. 2013;3:171-8.

6. Bossis G, Sarry J-E, Kifagi C, Ristic M, Saland E, Vergez F, et al. The ROS/ SUMO axis contributes to the response of acute myeloid leukemia cells to chemotherapeutic drugs. Cell Rep. 2014;7(6):1815-23.

7. Mochizuki N, Shimizu S, Nagasawa T, Tanaka H, Taniwaki M, Yokota J, et al. A novel gene, MEL1, mapped to 1 p36.3 is highly homologous to the MDS1-EVI1 gene and is transcriptionally activated in t(1 3)(p36 q21)-positive leukemia cells. Blood. 2000;96(9):3209-14.

8. Di Zazzo E, De Rosa C, Abbondanza C, Moncharmont B. PRDM Proteins: Molecular Mechanisms in Signal Transduction and Transcriptional Regulation. Biology. 2013;2(1):107-41.

9. Nishikata I, Sasaki H, Iga M, Tateno Y, Imayoshi S, Asou N, et al. A novel EVI1 gene family, MEL1, lacking a PR domain (MEL1S) is expressed mainly in $\mathrm{t}(1 ; 3)(\mathrm{p} 36 ; \mathrm{q} 21)$-positive AML and blocks G-CSF-induced myeloid differentiation. Blood. 2003;102(9):3323-32.

10. Shing DC, Trubia M, Marchesi F, Radaelli E, Belloni E, Tapinassi C, et al. Overexpression of sPRDM16 coupled with loss of p53 induces myeloid leukemias in mice. J Clin Invest. 2007;117(12):3696-707.

11. Bjork BC, Turbe-Doan A, Prysak M, Herron BJ, Beier DR. Prdm16 is required for normal palatogenesis in mice. Hum Mol Genet. 2010;19(5):774-89.

12. Aguilo F, Avagyan S, Labar A, Sevilla A, Lee D-F, Kumar P, et al. Prdm16 is a physiologic regulator of hematopoietic stem cells. Blood. 2011;117(19):5057-66. 
13. Chuikov1 S, Levi1 BP, Smith ML, Morrison SJ. Prdm16 promotes stem cell maintenance in multiple tissues, partly by regulating oxidative stress. Nat Cell Biol. 2010;12(10):999-1006.

14. Seale1 P, Bjork B, Yang W, Kajimura S, Chin S, Kuang S, et al. PRDM16 controls a brown fat/skeletal muscle switch. Nature. 2008;454(7207):961-7.

15. Kajimura S, Seale1 P, Kubota K, Lunsford E, Frangioni JV, Gygi SP, et al. Initiation of myoblast to brown fat switch by a PRDM16-C/EBP-beta transcriptional complex. Nature. 2009;460(7259):1154-8.

16. Villanueva CJ, Vergnes L, Wang J, Drew BG, Hong C, Tu Y, et al. Adipose subtype-selective recruitment of TLE3 or Prdm16 by PPARgamma specifies lipid storage versus thermogenic gene programs. Cell Metab. 2013;17(3):423-35

17. Ohno1 H, Shinoda1 K, Ohyama1 K, Sharp LZ, Kajimura S. EHMT1 controls brown adipose cell fate and thermogenesis through the PRDM16 complex. Nature. 2013;504(7478):163-7.

18. Duhoux FP, Ameye G, Montano-Almendras CP, Bahloula K, Mozziconacci MJ, Laibe $\mathrm{S}$, et al. PRDM16 (1p36) translocations define a distinct entity of myeloid malignancies with poor prognosis but may also occur in lymphoid malignancies. Br J Haematol. 2012;156(1):76-88.

19. Geiss-Friedlander R, MelchiorF. Concepts in sumoylation: a decade on. Nat Rev Mol Cell Biol. 2007;8(12):947-56.

20. Hickey CM, Wilson NR, Hochstrasser M. Function and regulation of SUMO proteases. Nat Rev Mol Cell Biol. 2012;13(12):755-66.

21. Sarge 1 KD, Park-Sarge O-K. Sumoylation and human disease pathogenesis. Trends Biochem Sci. 2009:34(4):200-5.

22. Nishikata I, Nakahata S, Saito Y, Kaneda K, Ichihara E, Yamakawa N, et al. Sumoylation of MEL1S at lysine 568 and its interaction with CtBP facilitates its repressor activity and the blockade of G-CSF-induced myeloid differentiation. Oncogene. 2011;30(40):4194-207.

23. Cheng J, Kang X, Zhang S, Yeh ETH. SUMO-Specific Protease 1 Is Essential for Stabilization of HIF1a during Hypoxia. Cell. 2007;131(3):584-95.

24. Li MJ, Rossi JJ. Lentivirus Transduction of Hematopoietic Cells. Cold Spring Harb Protoc. 2007;2007(5):pdb.prot4755.

25. Duan C-W, Shi J, Chen J, Wang B, Yu Y-H, Qin X, et al. Leukemia propagating cells rebuild an evolving niche in response to therapy. Cancer Cell. 2014:25(6):778-93.

26. Harms MJ, Ishibashi J, Wang W, Lim H-W, Goyama S, Sato T, et al. Prdm16 is required for the maintenance of brown adipocyte identity and function in adult mice. Cell Metab. 2014;19(4):593-604.

27. Morishita K. Leukemogenesis of the EVI1/MEL1 gene family. Int J Hematol. 2007;85(4):279-86

28. Yoshida M, Nosaka K, Yasunaga J, Nishikata I, Morishita K, Matsuoka M. Aberrant expression of the MEL1S gene identified in association with hypomethylation in adult T-cell leukemia cells. Blood. 2004;103(7):2753-60.

29. Pinheiro I, Margueron R, Shukeir N, Eisold M, Fritzsch C, Richter FM, et al. Prdm3 and Prdm16 are H3K9me1 methyltransferases required for mammalian heterochromatin integrity. Cell. 2012;150(5):948-60.

30. van Waalwijk van Doorn-Khosrovani SB, Erpelinck C, van Putten WLJ, Valk PJM, van der Poel-van de Luytgaarde S, Hack R, et al. High EVI1 expression predicts poor survival in acute myeloid leukemia: a study of 319 de novo AML patients. Blood. 2003;101(3):837-45.

31. Neyret-Kahn H, Benhamed M, Ye T, Le Gras S, Cossec J-C, Lapaquette P, et al. Sumoylation at chromatin governs coordinated repression of a transcriptional program essential for cell growth and proliferation. Genome Res. 2013;23(10):1563-79.

32. Singh S, Pradhan AK, Chakraborty S. SUMO1 negatively regulates the transcriptional activity of EVI1 and significantly increases its co-localization with EVI1 after treatment with arsenic trioxide. Biochimica et Biophysica Acta (BBA) - Molecular Cell Research. 2013;1833(10):2357-68.

33. Zhang W, Wang X, Xia X, Liu X, Suo1 S, Guo J, et al. Klf10 inhibits IL-12p40 production in macrophage colony-stimulating factor-induced mouse bone marrow-derived macrophages. Eur J Immunol. 2013:43(1):258-69.

34. Kreise D, Sugimoto S, Tietjens J, Zhu J, Yamamoto S, Krupnick AS, et al. Bcl3 prevents acute inflammatory lung injury in mice by restraining emergency granulopoiesis. J Clin Invest. 2011;121(1):265-76.

35. Yuan Z, Peng L, Radhakrishnan R, Seto E. Histone Deacetylase 9 (HDAC9) Regulates the Functions of the ATDC (TRIM29) Protein. Journal of Biological Chemistry. 2010;285(50):39329-38.

36. Hussen J, Frank C, Düvel A, Koy M, Schuberth H-J. The chemokine CCL5 induces selective migration of bovine classical monocytes and drives their differentiation into LPS-hyporesponsive macrophages in vitro. Developmental \& Comparative Immunology. 2014;47(2):169-77.

37. Gulino A, Di Marcotullio L, Screpanti I. The multiple functions of Numb. Exp Cell Res. 2010;316(6):900-6.

38. Subramaniam M, Hawse JR, Rajamannan NM, Ingle JN, Spelsberg TC. Functional role of KLF10 in multiple disease processes. Biofactors. 2010;36(1):8-18

39. Pece S, Confalonieri S, Romano PR, Di Fiore PP. NUMB-ing down cancer by more than just a NOTCH. Biochimica et Biophysica Acta (BBA) - Reviews on Cancer. 2011;1815(1):26-43.

\section{Submit your next manuscript to BioMed Central and take full advantage of:}

- Convenient online submission

- Thorough peer review

- No space constraints or color figure charges

- Immediate publication on acceptance

- Inclusion in PubMed, CAS, Scopus and Google Scholar

- Research which is freely available for redistribution 